\title{
Obtaining Informed Consent Using Patient Specific 3D Printing Cerebral Aneurysm Model
}

\author{
Pil Soo Kim, Chang Hwa Choi, In Ho Han, Jung Hwan Lee, Hyuk Jin Choi, Jae Il Lee \\ Department of Neurosurgery and Medical Research Institute, Pusan National University Hospital, Pusan National University School of Medi- \\ cine, Busan, Korea
}

Objective : Recently, three-dimensional (3D) printed models of the intracranial vascular have served as useful tools in simulation and training for cerebral aneurysm clipping surgery. Precise and realistic 3D printed aneurysm models may improve patients' understanding of the 3D cerebral aneurysm structure. Therefore, we created patient-specific 3D printed aneurysm models as an educational and clinical tool for patients undergoing aneurysm clipping surgery. Herein, we describe how these 3D models can be created and the effects of applying them for patient education purpose.

Methods : Twenty patients with unruptured intracranial aneurysm were randomly divided into two groups. We explained and received informed consent from patients in whom 3D printed models-(group I) or computed tomography angiography-(group II) was used to explain aneurysm clipping surgery. The 3D printed intracranial aneurysm models were created based on time-offlight magnetic resonance angiography using a 3D printer with acrylonitrile-butadiene-styrene resin as the model material. After describing the model to the patients, they completed a questionnaire about their understanding and satisfaction with aneurysm clipping surgery.

Results : The 3D printed models were successfully made, and they precisely replicated the actual intracranial aneurysm structure of the corresponding patients. The use of the 3D model was associated with a higher understanding and satisfaction of preoperative patient education and consultation. On a 5-point Likert scale, the average level of understanding was scored as 4.7 (range, 3.0-5.0) in group I. In group II, the average response was 2.5 (range, 2.0-3.0).

Conclusion : The 3D printed models were accurate and useful for understanding the intracranial aneurysm structure. In this study, 3D printed intracranial aneurysm models were proven to be helpful in preoperative patient consultation.

Key Words : Informed consent · Printing, Three-dimensional · Intracranial aneurysm.

\section{INTRODUCTION}

In the surgical treatment of unruptured intracranial aneurysms (UIAs), a thorough understanding of the positional re- lationship of the aneurysm with the parent artery and its branches, bones, brain parenchyma, and cranial nerves is of great importance ${ }^{6}$. In the past, surgical treatment was acquired through practical experience gained during actual op-

- Received : April 10, 2019 •Accepted : April 22, 2019

- Address for reprints : Jae II Lee

Department of Neurosurgery, Pusan National University Hospital, Pusan National University School of Medicine, 179 Gudeok-ro, Seo-gu, Busan 49241, Korea Tel : +82-51-240-7257, Fax : +82-51-244-0282, E-mail : medifirst@pusan.ac.kr, ORCID : https://orcid.org/0000-0003-1412-4146 
erations. Recently, however, there have been increasingly fewer opportunities for junior surgeons to receive on-the-job training owing to restrictions imposed by society and the extensive use of endovascular techniques ${ }^{5)}$.

To resolve this problem, three-dimensional (3D) printing technology has been adopted as neurosurgical training in recent years ${ }^{3,11,12)}$. With rapid advances in additive manufacturing and 3D printing ${ }^{1}$, 3D models of any organ can now be created directly from two-dimensional (2D) computed tomography scans or magnetic resonance images ${ }^{9}$. These models are physiologically and anatomically accurate and very helpful for gaining an understanding of the structures. For these reasons, these models are recently being used in the clinical setting, and they have shown to be effective teaching tools for training junior surgeons. The application of $3 \mathrm{D}$ printing technology in neurosurgical training has been reported in recent years and its usefulness has been demonstrat$\mathrm{ed}^{5,8,10)}$.

Such advantages led us to postulate that patient-specific 3D printed aneurysm models might be useful educational tools for patients and family members. Until now, 2D imaging, such as computed tomography angiography (CTA) or cerebral angiography, was the only method available for patient educations. With the digitalization of diagnostic radiologic images, it has become easy to obtain simulated 3D images at desired angles by, for example, 3D-CTA or 3D digital subtraction angiography (3D-DSA $)^{2}$. Although this method is useful, it is difficult to understand the complexity of the vascular network around the aneurysm, and all these images can only be presented on a computer screen.

We believe a true 3D printed model is more advantageous as an educational tools. However, no study has focused on patients' understanding and satisfaction of aneurysm clipping surgery. Thus, we created patient-specific 3D printed aneurysm models as an educational tool for patients who were scheduled to undergo aneurysm clipping surgery. In this study, we describe how these 3D models were created and the effects of applying them for patient education purposes.

\section{MATERIALS AND METHODS}

\section{Patients}

This prospective study included 20 consecutive patients with UIAs, who were admitted to Neurosurgery from November 2017 to August 2018. All patients included in this study underwent aneurysm clipping surgery that was performed by a single surgeon in our department. The study was approved by the Pusan National University Hospital Institutional Review Board (1811-008-072).

We explained the study and received informed consent from all patients. The 20 patients with UIAs were randomly divided into two groups. The 3D printed models were used in 10 patients (group I) (Table 1) and CTA was used in the other 10 patients (group II) (Table 2). The 20 patients received similar information regarding the anatomy and physiology of the aneurysm, the planned surgery, and associated surgical risks and outcomes from single doctor, except for differences in explanatory material.

\section{Preparation of magnetic resonance data for 3D printing}

Aneurysm models were fabricated using data obtained from time-of-flight magnetic resonance angiography (MRA) data (Fig. 1). MRA data of all enrolled patients were prospectively collected using a 1.5 Tesla MAGNETOM Avanto scanner (Siemens Healthineers, Erlangen, German). We used Materialise Mimics software (Materialise, Leuven, Belgium) to process the original MRA data in Digital Image and Communication in Medicine (DICOM) format, and the vascular threshold values were applied for patients with an aneurysm detected by MRA. A 3D reconstruction technique was used to construct a 3D intracranial aneurysm model. The results were converted to a standard triangulated language file (Fig. 2).

\section{D printing}

The 3D printed models were created with Nobel 1.0A (XYZPrinting, San Diego, CA, USA) (Fig. 3). This printer works with full-color ink-jet printing on thin layers of plaster powder. It produces solid models by using photosensitive resin to repeatedly draw $0.025-\mathrm{mm}$ thick pattern layers on a platform. The solid model is hard and whitish. The model is formed together with automatically generated supports. The supports have to be removed after the model is taken off the platform.

\section{Data collection}

Clinical data of the patients, including age, sex, academic 
Table 1. Clinical data of group I

\begin{tabular}{lccccc}
\hline Patient No. & Sex & Age (years) & Location of the lesion & Size $(\mathbf{m m}), \mathbf{L} \times \mathbf{W} \times \mathbf{H}$ & Diameter of the neck $(\mathbf{m m})$ \\
\hline 1 & F & 54 & Rt ICA bifurcation & $2.82 \times 4.18 \times 2.76$ & 1.83 \\
2 & F & 58 & Rt MCA bifurcation & $4.04 \times 4.69 \times 5.13$ & 3.15 \\
3 & F & 61 & Rt MCA bifurcation & $4.50 \times 6.28 \times 5.80$ & 3.32 \\
4 & F & 57 & Lt A-com & $6.11 \times 4.95 \times 6.97$ & 2.62 \\
\hline 5 & F & 71 & Rt MCA bifurcation & $8.06 \times 7.19 \times 7.84$ & 4.28 \\
\hline 6 & M & 50 & Rt M1 & $3.33 \times 3.79 \times 4.55$ & 2.86 \\
\hline 8 & F & 62 & Lt M2 & $5.38 \times 6.02 \times 5.43$ & 3.39 \\
9 & F & 54 & Rt MCA bifurcation & $4.32 \times 3.96 \times 5.28$ & 3.21 \\
\hline 10 & F & 58 & Rt MCA bifurcation & $3.87 \times 4.03 \times 3.42$ & 3.38 \\
\hline
\end{tabular}

No. : number, L : length, $\mathrm{W}$ : width, $\mathrm{H}$ : height, $\mathrm{F}$ : female, Rt : right, ICA : internal carotid artery, MCA : middle cerebral artery, Lt : left, A-com : anterior communicating artery, $\mathrm{M}$ : male, Ach : anterior choroidal artery

Table 2. Clinical data of group II

\begin{tabular}{lccccc}
\hline Patient No. & Sex & Age (years) & Location of the lesion & Size $(\mathbf{m m}), \mathbf{L} \times \mathbf{W} \times \mathbf{H}$ & Diameter of the neck $(\mathbf{m m})$ \\
\hline 1 & F & 53 & Lt P-com & $6.44 \times 6.01 \times 3.39$ & 4.49 \\
2 & F & 68 & Lt MCA bifurcation & $8.25 \times 5.39 \times 8.11$ & 5.56 \\
3 & M & 70 & Rt P-com & $3.36 \times 2.69 \times 3.79$ & 2.39 \\
4 & F & 58 & Rt A-com & $5.10 \times 4.48 \times 4.95$ & 4.31 \\
5 & M & 57 & Lt A-com & $4.93 \times 6.85 \times 5.88$ & 3.39 \\
6 & F & 56 & Lt MCA bifurcation & $6.25 \times 4.58 \times 4.97$ & 5.12 \\
\hline 7 & M & 63 & Rt M1 & $4.38 \times 3.56 \times 5.92$ & 3.32 \\
9 & F & 64 & Rt MCA bifurcation & $5.93 \times 5.82 \times 4.57$ & 6.78 \\
\hline 10 & F & 59 & Rt A-com & $4.42 \times 5.28 \times 5.42$ & 3.82 \\
\hline
\end{tabular}

No. : number, L : length, W : width, H : height, F : female, Lt : left, P-com : posterior communicating artery, MCA : middle cerebral artery, M : male, Rt : right, A-com : anterior communicating artery
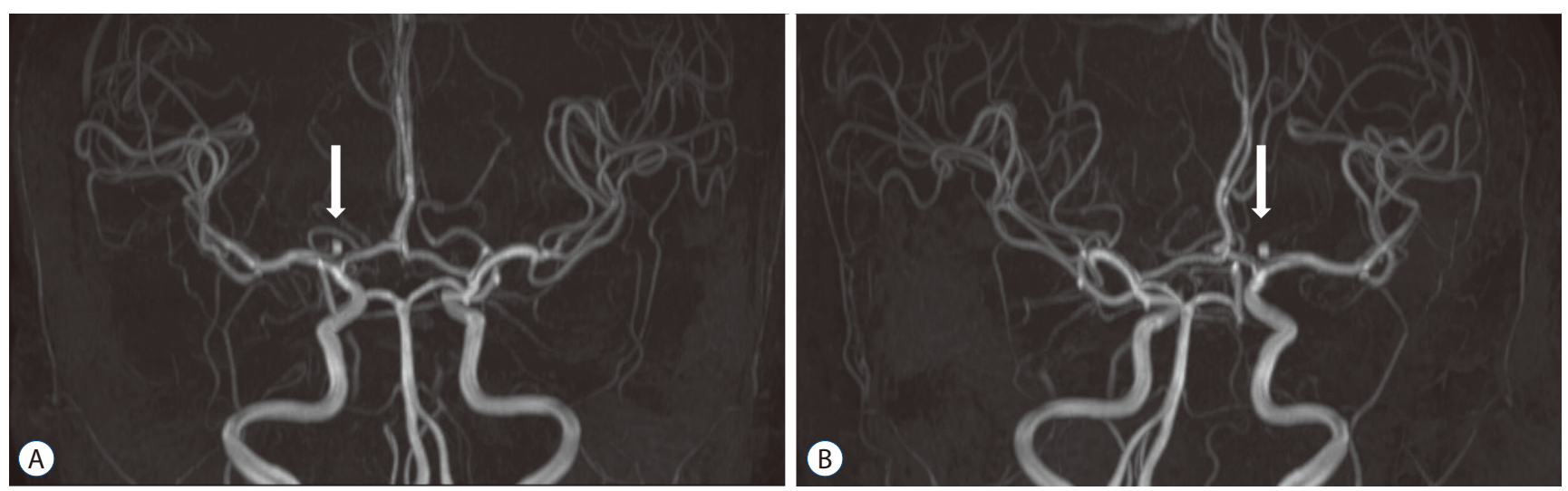

Fig. 1. Magnetic resonance angiography (MRA) of a right internal carotid artery bifurcation aneurysm before surgery. White arrows showed aneurysms on MRA. A : Anteroposterior view. B : Posteroanterior view. 
background, location of the aneurysm, lumen size of the aneurysm, and diameter of the aneurysm neck, were recorded.

\section{Questionnaire for patient consultation}

We assessed patients' understanding and satisfaction of the presurgical consultation with the 3D models using a questionnaire. The questionnaire consisted of seven questions, and ratings were provided on 5-point Likert scale.

\section{RESULTS}

In this study, 20 patients with UIAs (five men and 15 women; mean age, 59.4 years [range, 50-71]) were enrolled. Characteristics of age and level of education were similar between the two groups. The location, size, and diameter of aneurysm were summarized in Tables 1 and 2 for each group. There are no statistically significant differences in the mean size of the aneurysm which length (group I, $4.56 \pm 1.57 \mathrm{~mm}$; group II, 5.60 $\pm 1.57 \mathrm{~mm} ; p=0.144$ ), width (group I, $5.01 \pm 1.14 \mathrm{~mm}$; group II, $5.06 \pm 1.25 \mathrm{~mm} ; p=0.924$ ), and height (group I, $5.12 \pm 1.54 \mathrm{~mm}$;
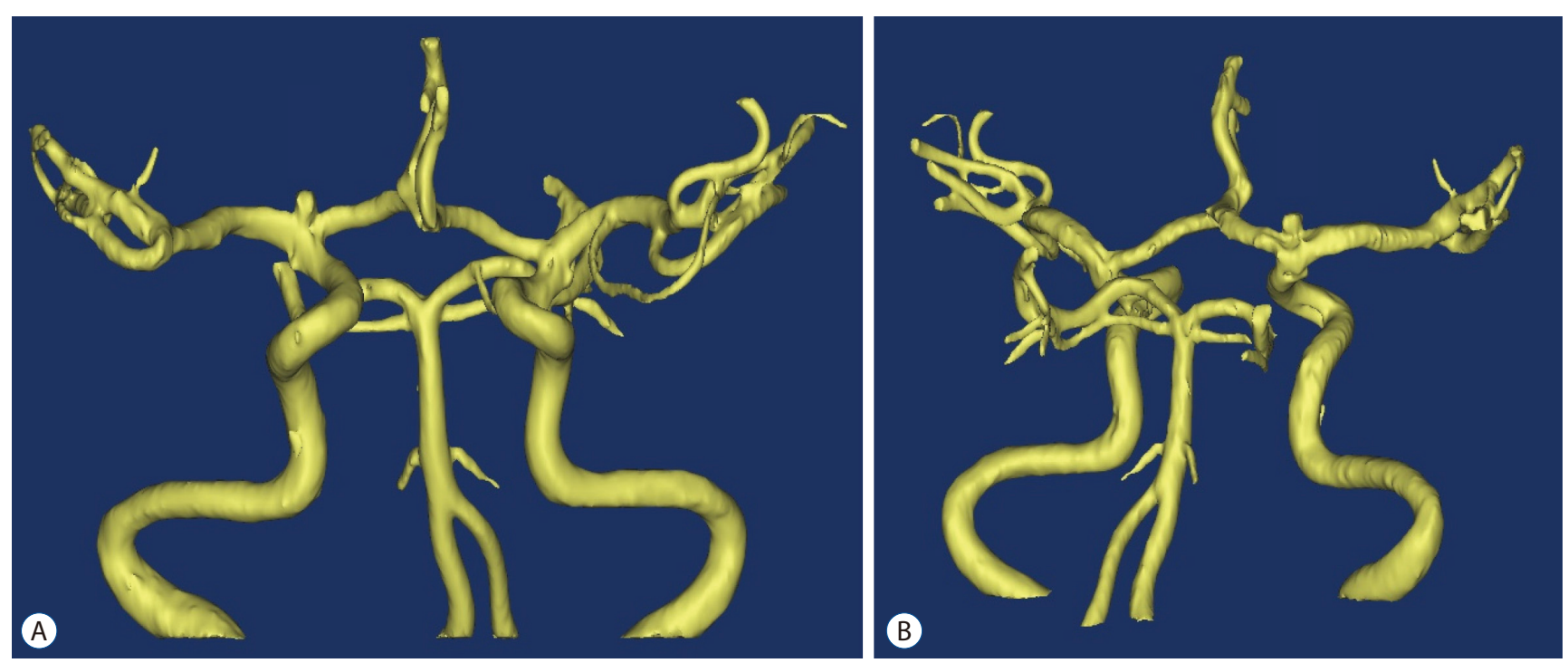

Fig. 2. Three-dimensional reconstruction of a right internal carotid artery bifurcation aneurysm before surgery using Materialise Mimics software (Materialise, Leuven, Belgium). A : Anteroposterior view. B : Posteroanterior view.
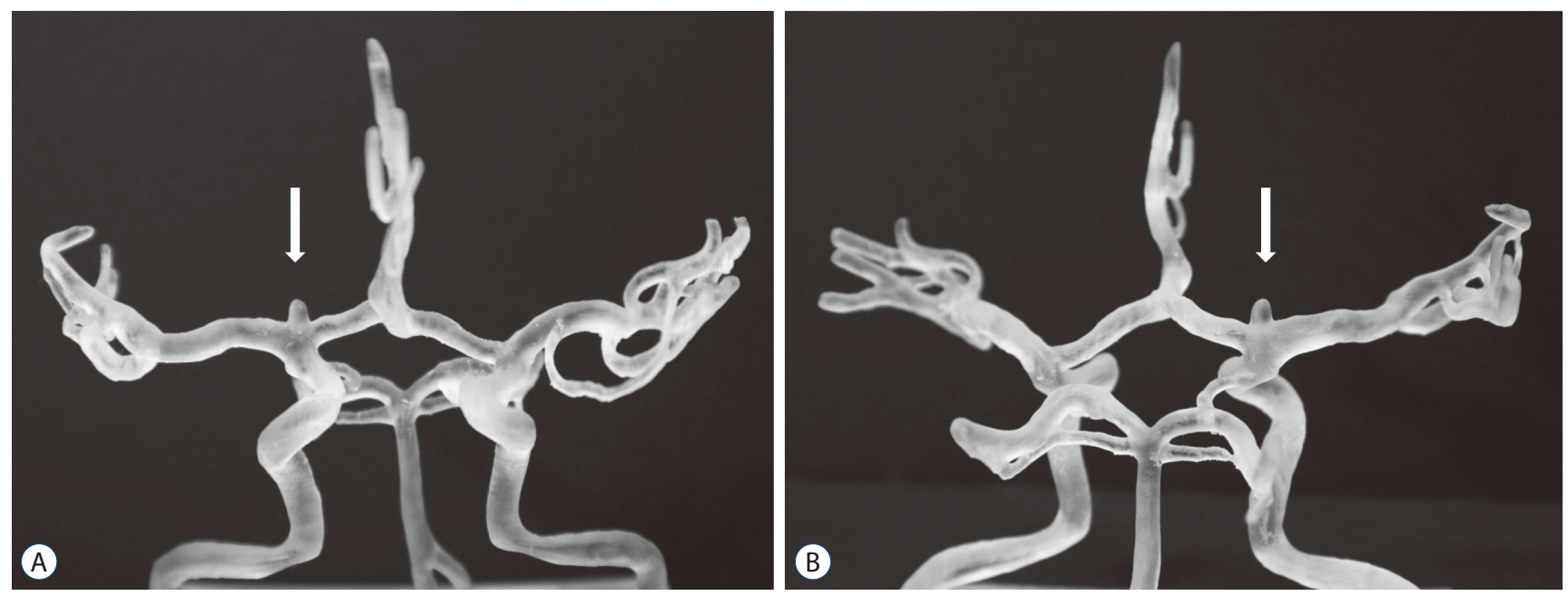

Fig. 3. The three-dimensional printed model of an internal carotid artery bifurcation aneurysm before surgery. White arrows demonstrated aneurysms on 3D model. A : Anteroposterior view. B : Posteroanterior view. 
group II, $5.61 \pm 1.78 \mathrm{~mm} ; p=0.524)$ between two groups. The mean diameter of aneurysm neck of group I was $3.43 \pm 0.55$ $\mathrm{mm}$, whereas the mean diameter of group II was $4.11 \pm 0.94$ $\mathrm{mm}(p=0.063)$.

All patients underwent aneurysm clipping surgery under general anesthesia. Additionally, all 20 patients underwent CTA, DSA, and MRA, and the 3D models of the aneurysms were printed based on MRA DICOM data. The proposed method successfully produced patient-specific 3D printed aneurysm models that precisely matched the images reconstructed through 3D DSA and Materialise Mimics. A total of 20 patients in group I and II participated in the survey after completing the consultation. Table 3 shows the questions of the questionnaire with the mean responses and ranges in group I and II.

The time required for manufacturing was 3-4.5 hours, and it tended to increase with the size of the 3D vascular model. This was mainly due to the longer time required to manufacture the 3D model. The process time and cost of the material are shown in Table 4.

Table 5 shows the levels of understanding and satisfaction both before and after the explanation in group I and group II. On average, the level of understanding scores improved from $1.7 \pm 0.67$ to $4.7 \pm 0.67$ in group I, and from $1.5 \pm 0.53$ to $2.5 \pm 0.53$ in group II. The level of satisfaction scores were $4.4 \pm 0.70$, and $2.9 \pm 0.57$ in group I and II, respectively (Table 5). The 3D models provided a better understanding of UIAs and aneurysm clipping surgery with a high level of patient satisfaction.

\section{DISCUSSION}

This current study investigated the impact of using 3D printed aneurysm models of the patient for patient education compared with CTA. In order to reduce the bias that could arise from differences in the description rather than differences in the explanatory material, single doctor gave an explanation for the patients. The 3D model was used as an adjunct to the conventional preoperative consent process. In the current study, it was possible to quantify the impact of the $3 \mathrm{D}$ models on patient understanding. Compared with conventional methods, the results suggested that 3D printed intracranial vascular models comprehensively improved patients' understanding and satisfaction of UIAs and clipping surgery.

Currently, the use of $3 \mathrm{D}$ printing in medicine is rapidly

Table 4. The process time and cost of the 3D printed models

\begin{tabular}{lcc}
\hline Patient No. & Process time (hours) & Cost (KRW) \\
\hline 1 & 3.5 & 3000 \\
2 & 3 & 2800 \\
3 & 4 & 3600 \\
4 & 3.5 & 3500 \\
5 & 4 & 4000 \\
6 & 4.5 & 4200 \\
7 & 4 & 3800 \\
8 & 3 & 3400 \\
9 & 4 & 3800 \\
10 & 3.5 & 3500 \\
\hline
\end{tabular}

3D : three-dimensional, No. : number, KRW : Korean won

Table 3. Results of the survey in group I and group II

\begin{tabular}{|c|c|c|c|}
\hline \multirow{2}{*}{ No. } & \multirow{2}{*}{ Question } & \multicolumn{2}{|c|}{ Average response (range, $1-5$ ) } \\
\hline & & Group I & Group II \\
\hline 1 & Did you know about the aneurysms before? & $1.7(1-3)$ & $1.5(1-2)$ \\
\hline 2 & $\begin{array}{l}\text { Did the 3D printed model improve your understanding of the aneurysm's relationship to the parent } \\
\text { artery? }\end{array}$ & $4.2(3-5)$ & NA \\
\hline 3 & Was the 3D printed model effective in the consultation? & $4.4(4-5)$ & NA \\
\hline 4 & $\begin{array}{l}\text { Did the 3D printed model improve your understanding of the intracranial aneurysm and aneurysm } \\
\text { clipping surgery? }\end{array}$ & $4.7(3-5)$ & $2.5(2-3)$ \\
\hline 5 & Were you satisfied with the consultation? & $4.4(3-5)$ & $2.9(2-4)$ \\
\hline 6 & Was the 3D printed model interesting to you? & $4.4(4-5)$ & NA \\
\hline 7 & Do you think the 3D printed model can be clinically applied to another pretreatment method? & $4(3-5)$ & NA \\
\hline
\end{tabular}

A score of 1 is strongly negative, and a score of 5 is strongly positive. No. : number, 3D : three-dimensional, NA : not available 
Table 5. Results of the survey in group I and group II

\begin{tabular}{|c|c|c|c|}
\hline Factor & $\begin{array}{c}\text { Group I, } \\
\text { average response }\end{array}$ & $\begin{array}{l}\text { Group II, } \\
\text { average response }\end{array}$ & $p$-value \\
\hline Age (years) & $57.9 \pm 5.8$ & $60.9 \pm 5.4$ & 0.247 \\
\hline $\operatorname{Sex}(F / M)(\%)$ & $90 / 10$ & $60 / 40$ & 0.280 \\
\hline Level of education & & & 0.853 \\
\hline None/lower education & $0(0)$ & $0(0)$ & \\
\hline High school graduate & $1(10)$ & $2(20)$ & \\
\hline Middle school graduate & $4(40)$ & $3(30)$ & \\
\hline College graduate & $5(50)$ & $5(50)$ & \\
\hline Level of understanding before the explanation & $1.7 \pm 0.67$ & $1.5 \pm 0.53$ & 0.579 \\
\hline Level of understanding after the explanation & $4.7 \pm 0.67$ & $2.5 \pm 0.53$ & $<0.001$ \\
\hline Level of satisfaction about the explanation & $4.4 \pm 0.70$ & $2.9 \pm 0.57$ & $<0.001$ \\
\hline
\end{tabular}

Values are presented as mean \pm standard deviation or number (\%) unless otherwise indicated. Level of understanding and level of satisfaction scores are based on a 5-point Likert scale. Age, level of understanding, and level of satisfaction scores are presented as averages. F : female, M : male

growing in many surgical fields but mostly in surgical training and simulation ${ }^{2,35}$. The use of 3D printing technology is rapidly expanding, not only in surgical training and simulation, but also in the area of medical education for patients ${ }^{4}$. We successfully created patient specific 3D models to inform patients with UIA about their clinical situation, treatment options, and risk. The models were highly appreciated by the patients. Patients significantly improved their understanding of all aspects investigated with the $3 \mathrm{D}$ printed models compared with conventional methods. Observing a physical representation of the aneurysm did not make patients feel uncomfortable. The use of a 3D printed model herein gave patients a much better idea of what would happen during the surgery, which reassured them, and consequently, the patients felt more involved in the decision-making process.

The main limitation of 3D printing technology is that building a 3D model involves a 3D rendering and 3D printing, which are time consuming and costly ${ }^{7)}$. However, with the development of newer printers and the broadening of printed materials, the time and cost have been reduced ${ }^{13)}$. In this study, we used the Nobel 1.0A (XYZPrinting), which is small $(280 \times 345 \times 590 \mathrm{~mm})$ and lightweight $(9.6 \mathrm{~kg})$. The process time for making the $3 \mathrm{D}$ models is 3.7 hours on average. The material cost of each model is approximately 3500 Korea won. The time and cost will decrease as 3D printing technology advances. As 3D printing technology progresses and costs fall, patient-specific 3D printing may become standard for both clinical and educational purposes.
This study had several limitations. The model integrated only the vascular structure with the aneurysm. Other important anatomical relationships such as the other optic chiasm, cranial nerves, skulls, and brains were not included. In addition, the 3D printing technology could capture only major vasculature structures. It is not possible to capture small vessels and perforators with current commercially-available technology.

Furthermore, this study only enrolled 20 patients, and the small sample sizes may introduce bias. Given that this was only a single-center experience and that there were only 10 patient-specific models used to evaluate patient understanding, additional larger prospective studies will be needed to confirm the usefulness of a patient-specific 3D print model for patient understanding and satisfaction with aneurysm clipping surgery.

\section{CONCLUSION}

The patient-specific 3D printed aneurysm models were useful patient education. The models were accurate, realistic, and easy to understand, although they illustrated the complex intracranial vascular structure. Moreover, such models are easy to create, and we believe that they are very useful in routine clinical practice during pretreatment. However, studies with larger sample sizes are needed to confirm these preliminary findings. 


\section{CONFLICTS OF INTEREST}

No potential conflict of interest relevant to this article was reported.

\section{INFORMED CONSENT}

Informed consent was obtained from all individual participants included in this study.

\section{AUTHOR CONTRIBUTIONS}

\author{
Conceptualization : JIL \\ Data curation : PSK \\ Formal analysis : PSK \\ Funding acquisition : JHL \\ Methodology : IHH \\ Project administration : JIL \\ Visualization : JHL \\ Writing - original draft : PSK \\ Writing - review \& editing : CHC, JIL
}

\section{- Acknowledgements}

This work was supported by medical ICT Convergence center of Busan economic promotion agency.

\section{References}

1. Aoun RJ, Hamade YJ, Zammar SG, Patel NP, Bendok BR : Futuristic three-dimensional printing and personalized neurosurgery. World Neurosurg $84: 870-871,2015$
2. Dong $\mathrm{M}$, Chen $\mathrm{G}$, Li J, Qin K, Ding X, Peng C, et al. : Three-dimensional brain arteriovenous malformation models for clinical use and resident training. Medicine (Baltimore) 97 : e9516, 2018

3. Kimura T, Morita A, Nishimura K, Aiyama H, Itoh H, Fukaya S, et al. : Simulation of and training for cerebral aneurysm clipping with 3-dimensional models. Neurosurgery 65 : 719-725, 2009

4. Knoedler M, Feibus AH, Lange A, Maddox MM, Ledet E, Thomas R, et al. : Individualized physical 3-dimensional kidney tumor models constructed from 3-dimensional printers result in improved trainee anatomic understanding. Urology 85 : 1257-1261, 2015

5. Mashiko T, Kaneko N, Konno T, Otani K, Nagayama R, Watanabe E : Training in cerebral aneurysm clipping using self-made 3-dimensional models. J Surg Educ 74 : 681-689, 2017

6. Mashiko T, Otani K, Kawano R, Konno T, Kaneko N, Ito Y, et al. : Development of three-dimensional hollow elastic model for cerebral aneurysm clipping simulation enabling rapid and low cost prototyping. World Neurosurg 83 : 351-361, 2015

7. Mitsouras D, Liacouras P, Imanzadeh A, Giannopoulos AA, Cai T, Kumamaru KK, et al. : Medical 3D printing for the radiologist. Radiographics 35 : 1965-1988, 2015

8. Ploch CC, Mansi CSSA, Jayamohan J, Kuhl E : Using 3D printing to create personalized brain models for neurosurgical training and preoperative planning. World Neurosurg 90 : 668-674, 2016

9. Rengier $F$, Mehndiratta A, von Tengg-Kobligk $H$, Zechmann CM, Unterhinninghofen R, Kauczor HU, et al. : 3D printing based on imaging data: review of medical applications. Int J Comput Assist Radiol Surg 5 : 335-341, 2010

10. Ryan JR, Almefty KK, Nakaji P, Frakes DH : Cerebral aneurysm clipping surgery simulation using patient-specific 3D printing and silicone casting. World Neurosurg 88 : 175-181, 2016

11. Wurm G, Lehner M, Tomancok B, Kleiser R, Nussbaumer K : Cerebrovascular biomodeling for aneurysm surgery: simulation-based training by means of rapid prototyping technologies. Surg Innov 18 : 294-306, 2011

12. Wurm G, Tomancok B, Pogady P, Holl K, Trenkler J : Cerebrovascular stereolithographic biomodeling for aneurysm surgery. Technical note. J Neurosurg 100 : 139-145, 2004

13. Yang T, Tan T, Yang J, Pan J, Hu C, Li J, et al. : The impact of using threedimensional printed liver models for patient education. J Int Med Res 46 : 1570-1578, 2018 